\title{
Podoplanin Is Regulated by AP-1 and Promotes Platelet Aggregation and Cell Migration in Osteosarcoma
}

\author{
Akiko Kunita, ${ }^{*}$ Takeshi G. Kashima, ${ }^{* \dagger}$ \\ Atsushi Ohazama, ${ }^{\ddagger}$ Agamemnon E. Grigoriadis, ${ }^{\dagger}$ \\ and Masashi Fukayama* \\ From the Department of Pathology, * Graduate School of \\ Medicine, the University of Tokyo, Tokyo, Japan; the Department \\ of Craniofacial Development and Orthodontics, ${ }^{\dagger}$ King's College \\ London, Guy's Hospital, London, United Kingdom; and the \\ Department of Periodontics, ${ }^{*}$ Dental School, Showa University, \\ Tokyo, Japan
}

Podoplanin is a type-I transmembrane sialomucinlike protein, which is expressed in a wide range of cell types and is involved in platelet aggregation and tumor metastasis. Here, we investigated the function, regulation, and expression of podoplanin in osteosarcoma. Podoplanin expression was observed in three osteosarcoma cell lines (MG-63, HOS, and U-2 OS) with platelet aggregation-inducing ability, which was blocked by podoplanin small-interfering RNA or a neutralizing antibody. Overexpression of podoplanin in nonmetastatic Dunn osteosarcoma cells promoted cell migration without attenuating cell proliferation. Both podoplanin and TGF- $\beta 1$ were up-regulated by c-Fos induction in MC3T3-E1 osteoblastic cells, and were highly expressed in c-Fos transgenic mouse osteosarcomas and c-Fos-transformed osteosarcoma cell lines. Immunohistochemistry of human osteosarcoma tissue microarrays $(n=133)$ showed staining of tumor cells embedded in an excess of irregular neoplastic bone matrix in $100 \%$ of tumors undergoing so-called "normalization/maturation." Podoplanin was also expressed in osteosarcoma subtypes, with $65 \%$ of osteoblastic, $100 \%$ of chondroblastic, and $79 \%$ of fibroblastic tumors. CD44 and PERM immunohistochemistry showed coexpression with podoplanin in both mouse and human osteosarcoma. Podoplanin expression was significantly higher in metastatic osteosarcomas $(n=6)$ than in primary osteosarcomas $(n=10)$. Our data suggest that podoplanin, which is not expressed in normal osteoblasts but in osteocytes, is aberrantly expressed in transformed osteoblasts and in osteosarcoma, and is under AP-1 transcriptional control. Thus podoplanin is a candidate molecule for ther- apeutic targeting. (Am J Pathol 2011, 179:1041-1049; DOI: 10.1016/j.ajpath.2011.04.027)

Osteosarcoma (OS) is the most common primary malignant bone tumor, with a high tendency to metastasize to the lung. Despite recent advances in modern chemotherapy, the average survival after a recurrence in distant organs is less than 1 year. ${ }^{1}$ In contrast, of patients who present with no metastasis, approximately $70 \%$ will be long-term survivors. ${ }^{2}$ Therefore, there is a strong necessity to better understand the molecular mechanisms of metastasis to deliver innovative life-saving and life-enhancing therapies to patients.

Platelet aggregation is one of the crucial steps involved during the sequential tumor metastasis process to escape from the host immune system and form tumor emboli in distant organs. Several earlier studies have shown that platelet aggregating capability of tumor cells from colon cancer and melanoma is correlated with their metastatic potential in vivo. ${ }^{3-5}$ Podoplanin, a type-I transmembrane sialomucin-like glycoprotein, is a platelet aggregation-inducing factor ${ }^{6}$ that is expressed in a wide range of tumors ${ }^{7-9}$ and has been reported to be associated with poor outcome of oral and esophageal squamous cell carcinomas. ${ }^{10,11}$ Podoplanin promotes cell migration and cell invasion,, 12 and also plays a key role in epithelial-mesenchymal transition in Madin-Darby canine kidney (MDCK) type-II cells. ${ }^{13}$ These observations suggest that podoplanin contributes to the aggressive behavior of cancer cells during cancer progression. ${ }^{9}$ In-

Supported in part by a grant for Research Fellowships from the Japanese Society for the Promotion of Science for Young Scientists, Japan (A.K.), a grant (no 16790202 to T.G.K.) from the Ministry of Education, Culture, Sports, Science \& Technology, and a grant from the UK Bone Cancer Research Trust (No. 09/08 to A.E.G.).

Accepted for publication April 29, 2011.

Supplemental material for this manuscript can be found at http://ajp. amjpathol.org or at doi: 10.1016/j.ajpath.2011.04.027.

Current address of A.O., Department of Craniofacial Development, King's College London, Guy's Hospital, London, United Kingdom.

Address reprint requests to: Takeshi G. Kashima, M.D., Ph.D., Histopathology Department, Nuffield Orthopaedic Centre, Windmill Road, Oxford, OX3 7LD, United Kingdom. E-mail: kashima-tky@umin.net. 
deed, we have shown that ectopic expression of podoplanin induces tumor metastasis in vivo. ${ }^{14}$

Podoplanin has been known by various antigen nomenclatures including PA2.26, T1a, E11, OTS-8, gp36, gp38, gp40, and Aggrus, and is expressed in a wide range of normal cell types. ${ }^{8}$ In normal bone, in addition to lymphatics, ${ }^{15}$ podoplanin is expressed exclusively by osteocytes, especially newly formed and young osteocytes (osteoid osteocytes). Gain- and loss-of-function studies have demonstrated that podoplanin plays a functional role in the formation of osteocyte dendritic processes. ${ }^{16}$

Several growth factors, including epidermal growth factor (EGF), basic fibroblast growth factor (FGF2), and transforming growth factor- $\beta$ (TGF- $\beta$ ), are known to induce podoplanin expression in MCF7 breast cancer cells. ${ }^{12}$ In contrast, the transcriptional regulation of podoplanin expression is not well understood; only the Sp1/ Sp3 transcription factor, ${ }^{17}$ the $\mathrm{Src} / \mathrm{Cas}$ pathway, ${ }^{18}$ and the c-Fos proto-oncogene ${ }^{19}$ have been reported to modulate podoplanin expression so far. c-Fos is a member of the AP-1 transcription factor family, comprising heterodimers with members of the C-Jun and ATF. ${ }^{20,21}$ Extensive work using gain- and loss-of-function analyses in mice have established an important role for c-Fos in proliferation and differentiation of normal cells, but also in cellular transformation and tumor formation. ${ }^{19,22,23}$ Particularly, we have shown previously that deregulated c-Fos expression in vivo causes osteosarcomas (OS) in mice $^{24}$ and high levels of c-Fos expression have been observed in the vast majority of human OS. ${ }^{25}$

Although the role of podoplanin in platelet aggregation, cell migration, and metastasis of carcinoma cells is established, there has been one previous report in human OS cell lines suggesting that their ability to induce platelet aggregation in vitro might relate to their ability to metastasize. ${ }^{26}$ We therefore postulated that podoplanin expression would be expressed in human OS in vivo and involved in OS metastasis. To this end, we investigated the role of podoplanin on their platelet aggregation inducing activity as well as cell migration capacity in OS cells. Further, as a c-Fos target gene, we investigated the expression of podoplanin in C-Fos-inducible osteoblastic cell lines ${ }^{27}$ and in C-Fos transgenic mouse OS. ${ }^{24}$ Finally, we carried out detailed expression analysis of podoplanin in a number of human OS. Our results indicated a potential use of podoplanin for therapeutic aims.

\section{Materials and Methods}

\section{Cell Culture and Establishing Stable Podoplanin Overexpressing OS Cells}

The mouse OS cell line Dunn (a kind gift from Dr. Takafumi Ueda, Osaka University, Japan), the human OS cell lines (all obtained from American Type Culture Collection, Manassas, VA), MG-63, HOS, and U-2 OS, and the mouse OS cell lines P1.7, P1.9, and P1.15, derived from bone tumors formed in C-Fos-overexpressing transgenic mice, were cultured under standard conditions. ${ }^{24,28}$ The tetracycline-regulatable, c-Fos-overexpressing MC3T3-E1 mouse osteoblastic cell subclone, AT9.2, was cultured in the presence of tetracycline, and exogenous c-Fos expression was induced following withdrawal of tetracycline (Tetoff system). ${ }^{27}$ Normal human primary osteoblasts were purchased (Lonza, Basel, Switzerland) and used within a few passages. For establishment of stable podoplanin-overexpressing cells, transfection of pcDNA3-human podoplanin $\mathrm{CDNA}^{6}$ and a control pcDNA3 vector into Dunn cells was performed using LipofectAMINE 2000 reagent (Invitrogen, Carlsbad, CA), and the colonies showing resistance to G418 were isolated.

\section{Human Tissue Specimens}

We analyzed a series of primary OS $(n=100)$ and pulmonary metastases ( $n=33$ ) using tissue microarrays (TMAs) as well as four normal human bone sections. The samples were routinely fixed in 10\% formalin, decalcified, then embedded in paraffin. TMA blocks were constructed by using a manual tissue arrayer (Beecher Instrument, Sun Prairie, WI) using duplicated 2-mm cores for each case $(n=83)$. In addition, we also used a commercial OS TMA containing 50 single 1-mm cores from human OS (OS801; US Biomax, Rockville, MD).

First-strand cDNA samples were prepared from $0.5 \mu \mathrm{g}$ of total RNA as previously described. ${ }^{29}$ All samples were approved for use in this research by the Ethics Committee of the University of Tokyo (No.1220).

\section{Animals}

Ten tumors from five independent c-Fos transgenic mice (C57BL/6J strain, kindly provided by Dr. J.P. David, University of Erlangen, Germany) ${ }^{24}$ were examined by immunohistochemistry and in situ hybridization. Five-week-old BALB/c mice (Charles River Japan Inc., Kanagawa, Japan) were used for platelet aggregation assays. To generate xenograft samples, $10^{6}$ of $\mathrm{P} 1.15$ cells were subcutaneously injected into the back of nude mice (Charles River Japan Inc., Kanagawa, Japan), and the mice were sacrificed after 4 weeks $(n=4)$. The Animal Care and Use Committee and the Ethics Committee of the University of Tokyo approved the animal experiments described herein (1822T-020).

\section{Western Blot Analysis}

Total protein extracts from cultured cells and tissues were prepared and separated using SDS-PAGE and blotted as previously described. ${ }^{14}$ The membranes were incubated with an anti-human podoplanin antibody (NZ-1; AngioBio, Del Mar, CA) ${ }^{30}$ or an anti- $\beta$-actin antibody (Sigma, St. Louis, MO), followed by a horseradish peroxidase-conjugated secondary antibody, and developed using the ECL system (GE Healthcare, Chalfont St. Giles, Buckinghamshire, England, UK).

\section{RNA Interference}

Small-interfering RNA (siRNA) oligos, designed for specific podoplanin knockdown (hPDPN siRNA-sense: 
5'-GUCUGGCUUGACAACUCUGTT-3'; hPDPN siRNAantisense: 3'-CAGAGUUGUCAAGCCAGACTT-5'), were transiently introduced using LipofectAMINE 2000 reagent. As a negative control, we used a nonsilencing control siRNA. Cells were used for in vitro experiments 24 hours after siRNA transfection.

\section{Cell Migration Assay}

Cell migration assays were performed using transwell inserts ( $8 \mu \mathrm{m}$ pore size; Corning, NY), and the number of cells that migrated was counted after 18 hours as previously described. ${ }^{31}$ For antibody neutralization, $50 \mu \mathrm{g} / \mathrm{mL}$ of anti-human podoplanin antibody (NZ-1) or control normal rat IgG were mixed with cells, then incubated for 15 minutes on ice before seeding onto a transwell. All experiments were performed in triplicate.

\section{In Vitro Cell Proliferation Assay}

In vitro growth of stable transfectants was assessed using a Cell Counting Kit-8 (Dojin Laboratories, Kumamoto, Japan); the optical density was measured using a microplate reader (Model 550; Bio-Rad, Hercules, CA). To examine the threedimensional proliferation of stable clones, $10^{3}$ cells were seeded into a round-bottom 96-well plate (Sumilon Celltight Spheroid; Sumilon, Tokyo, Japan), and the mean diameters of the spheroids were measured every other day for 12 days as described previously. ${ }^{14}$

\section{Platelet Aggregation Assays}

Platelet aggregation was quantified by light transmittance using an MCM HEMA Tracer 313M (MC Medical, Tokyo, Japan). Platelet-rich plasma was prepared from fresh, heparinized blood that was drawn from BALB/c mice by cardiac puncture and then centrifuged $(170 \times g, 15$ minutes) at $23^{\circ} \mathrm{C}$. Platelet-poor plasma was obtained by centrifugation $(1500 \times g, 10$ minutes) of the remaining blood at $23^{\circ} \mathrm{C}$. Platelet-rich plasma was preincubated for 5 minutes at $37^{\circ} \mathrm{C}$, with constant stirring in the aggregometer. Platelet aggregation was then initiated by the addition of tumor cells $\left(1 \times 10^{6}\right.$ cells $\left./ \mathrm{mL}\right)$ and monitored as an extent of light transmittance to platelet-poor plasma. Alternatively, platelet aggregation was initiated by adding tumor cells $\left(5 \times 10^{6}\right.$ cells/sample $)$ and determined by measuring electric impedance, ${ }^{32}$ using a whole-blood aggregometer Model 560 (Chronolog, Havertown, PA).

\section{Semiquantitative RT-PCR and Quantitative Real-Time PCR}

The primers used in this study were followings: Mouse podoplanin (sense 5'-CTCAAGCTTCAAGATGTGGACCGTGCCAGT-3', antisense 5'-GAGGAATTCGGGCGAGAACCTTCCAGAAAT-'3), Mouse c-Fos (sense 5'-GCTGACAGATACACTCCAAGCGG-3', antisense 5'-AGGAAGACGTGTAAGTAGTGCAG-3'), Mouse Tgf $\beta 1$ (sense $5^{\prime}$ GACTCTCCACCTGCAAGACCA-3', antisense 5'-GGGACTGGCAGCCTTAGTT-3'), Mouse $\beta$-actin (sense 5'-GATA-
TCGCTGCGCTGGTCGTCGAC-3', antisense 5'-CAAGAAGGAAGGCTGGAAAAGAGC-3'), Human podoplanin (sense 5'-GGAAGGTGTCAGCTCTGCTC-3', antisense 5'CGCCTTCCAAACCTGTAGTC-3') and Human $\beta$-actin (sense 5'-ACTCTTCCAGCCTTCCTTCCTG-3', antisense 5'-ATCTCCTTCTGCATCCTGTCGG-3'). The first-strand cDNA were amplified by PCR, and real-time PCR was performed using QuantiTect SYBR Green PCR (Qiagen, Valencia, CA). Standard curves for templates of podoplanin and an endogenous control, $\beta$-actin, were generated by serial dilution of the PCR products $\left(1 \times 10^{8}\right.$ to $1 \times 10^{3}$ copies $\left./ \mathrm{mL}\right)$.

\section{Immunohistochemistry and in Situ Hybridization}

Sections were incubated with the following antibodies: anti-human podoplanin (NZ-1), anti-mouse podoplanin (RDI, Concord, MA), anti-human CD44 (GeneTex, Inc., Irvine, CA), anti-mouse CD44 (IM7; BD Pharmigen, Oxford, UK), anti-phospho-Ezrin (Thr567)/Radixin (Thr564)/ Moesin (Thr558) (Cell Signaling Technology Inc., Danvers, MA), anti-c-Fos (sc-52; Santa Cruz Biotechnology, Santa Cruz, CA), or anti-TGF- $\beta 1$ (sc-146; Santa Cruz Biotechnology). Sections for all of the antibodies, except NZ-1, were pre-treated using the microwave antigen retrieval method. After applying a biotin-conjugated secondary antibody, immunoreactivity was visualized using Vectastain ABC Kit (Vector Laboratories, Peterborough, UK). Assessment of immunohistochemistry was performed as described in Table 1. For detection of mouse Tgf- $\beta 1$, a Kpnl-Apal-digested fragment of the cDNA (approximately $600 \mathrm{bp}$, accession number: M13177) was labeled by ${ }^{35} \mathrm{~S}$ using in vitro transcription and in situ hybridization was carried out as described previously. ${ }^{33}$

\section{Statistical Analysis}

All data sets were analyzed using Graphpad Prism v.4 (Graphpad Software Inc., San Diego, CA) and shown as means \pm SEM. Mann-Whitney U-test, or one-way analysis of variance followed by Tukey-Kramer multiple comparisons was performed, where appropriate. $P$ values $<0.05$ were considered statistically significant. All statistical tests were two-tailed.

\section{Results}

\section{The Role of Podoplanin in Platelet Aggregation in OS Cell Lines}

We first addressed the function of podoplanin in OS by examining its ability to induce platelet aggregation. Three human OS cell lines, MG-63, HOS, and U-2 OS expressed high levels of endogenous podoplanin as examined by Western blotting (Figure 1A) and flow cytometry (see Supplemental Figure S1 at http://ajp.amjpathol.org). Incubation with platelet-rich plasma showed that podoplanin was functional, because all three OS cells were capable of inducing platelet aggregation (Figure 1B). To further confirm whether this platelet aggregation-inducing ability was depending on podoplanin, we knocked 
Table 1. Summary of Podoplanin Immunostaining of Human OS

\begin{tabular}{|c|c|c|c|}
\hline & ++ & + & - \\
\hline \multicolumn{4}{|l|}{ Primary tumors $(n=100)$} \\
\hline Normalization/maturation $(n=51 / 100)$ & $51(100 \%)$ & 0 & 0 \\
\hline \multicolumn{4}{|l|}{ Subtypes } \\
\hline Osteoblastic $(n=65 / 100)$ & $9(13.8 \%)$ & $30(46.1 \%)$ & $26(40 \%)$ \\
\hline Chondroblastic $(n=15 / 100)$ & $15(100 \%)$ & 0 & 0 \\
\hline Fibroblastic $(n=20 / 100)$ & $2(20 \%)$ & $13(65 \%)$ & $5(25 \%)$ \\
\hline \multicolumn{4}{|l|}{ Metastatic tumors $(n=33)$} \\
\hline Normalization/maturation $(n=15 / 33)$ & $15(100 \%)$ & 0 & 0 \\
\hline \multicolumn{4}{|l|}{ Subtypes } \\
\hline Osteoblastic $(n=15 / 33)$ & $5(33.3 \%)$ & $8(53.3 \%)$ & $2(13.3 \%)$ \\
\hline Chondroblastic $(n=4 / 33)$ & $4(100 \%)$ & 0 & 0 \\
\hline Fibroblastic $(n=14 / 33)$ & $2(14.3 \%)$ & $10(71.4 \%)$ & $2(14.3 \%)$ \\
\hline
\end{tabular}

A total of 133 osteosarcoma (OS) TMA samples (comprising 83 duplicated institutional and 50 single commercial cores) were examined by immunohistochemistry. Duplicated cores counted as one case. OS showing normalization/maturation ${ }^{34}$ were assessed for podoplanin expression at neoplastic dendritic processes. Membranous staining was also evaluated in each core, according to the conventional OS subtypes, osteoblastic, chondroblastic, and fibroblastic OS. Immunohistochemistry staining of TMA cores was evaluated semi-quantitatively based on number of positive tumor cells as follows:,$++>50 \%$ of tumor cells;,$+<50 \%$ of tumor cells; - , negative.

down podoplanin expression using a specific podoplanin siRNA, and Western blot analysis confirmed an $85 \%$ reduction in protein expression levels (Figure 1C). This resulted in a prolonged incubation period for stimulating platelet aggregation, suggesting a reduction in aggregating activity (Figure 1D). These observations indicate that podoplanin functions as a platelet aggregation-inducing factor in OS cells.
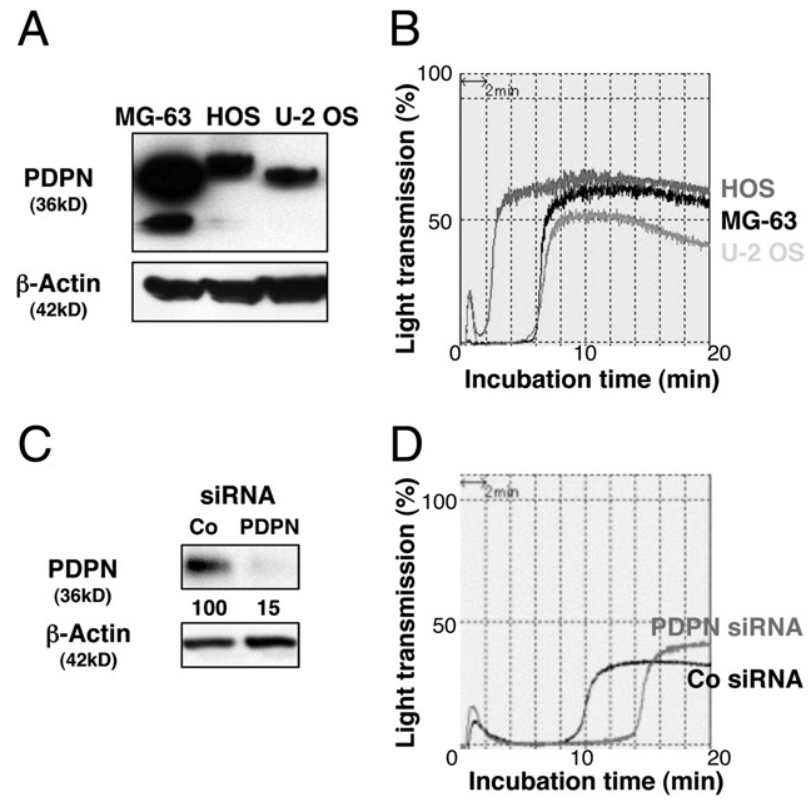

Figure 1. Podoplanin is expressed in human OS cell lines and promotes platelet aggregation. A: Western blot analysis showing that podoplanin is endogenously expressed in three human OS cell lines, MG-63, HOS, and U-2 OS. The different sized bands are due to differences in glycosylation patterns between the cell lines. B: The platelet aggregation-inducing capability of three human OS cell lines, MG-63, HOS, and U-2 OS was estimated by incubating the cells with mouse platelet-rich plasma, and was quantified by light transmittance. Platelet aggregation is induced in three human OS cell lines that express endogenous podoplanin. C: Podoplanin knockdown in OS cells. Control (Co) and podoplanin (PDPN) siRNA-transfected MG-63 cells were analyzed for podoplanin expression by Western blot. Numerical values represent podoplanin expression levels relative to $\beta$-actin quantified by densitometric analysis. D: Podoplanin siRNA inhibits platelet aggregation of MG-63 cells. Platelet aggregation was attenuated in podoplanin siRNA-transfected MG-63 cells
Next, we investigated the consequences of ectopic podoplanin expression using the mouse Dunn OS cell line that shows no endogenous expression of podoplanin protein. Three stable podoplanin-expressing clones (PDPN-11, -14, and -17) were used in further experiments. As shown in Figure 2A, Western blot analysis confirmed the expression level of exogenous podoplanin, where the expression level was the highest in PDPN-17, and PDPN-11 had the lowest expression. Cell surface expression of podoplanin was also confirmed by flow cytometry. We next investigated whether podoplanin overexpression promoted platelet aggregation in the transfectants. After 20 minutes, all of the podoplaninexpressing OS cells showed enhanced platelet aggrega-
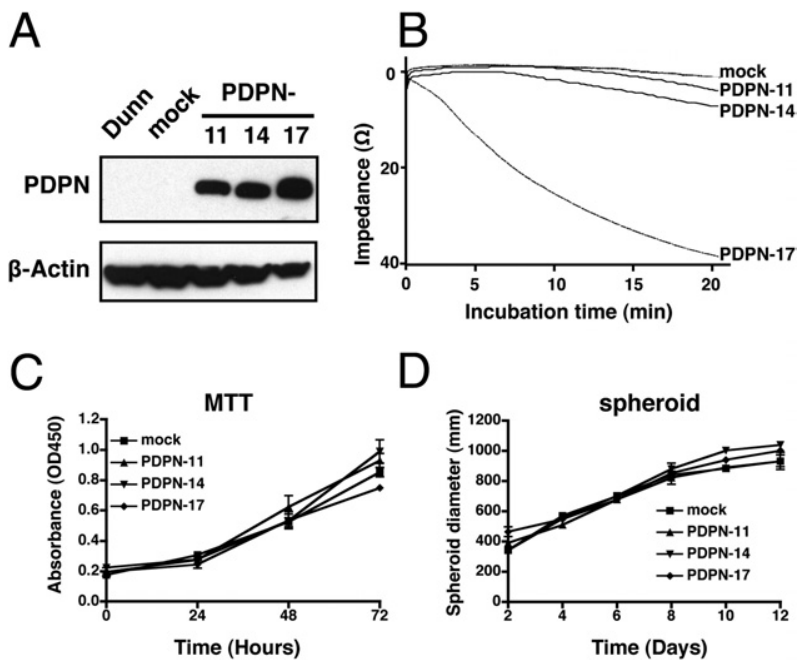

D

Figure 2. Behavior of podoplanin-overexpressing mouse Dunn OS cells. A: Overexpression of human podoplanin in stably transfected Dunn cells. Cell lysates from the Dunn/PDPN stable transfectants (PDPN-11, -14, and -17) and mock cells were subjected to Western blot analysis with an anti-podoplanin antibody. B: Platelet aggregation-inducing ability of the Dunn/PDPN stable transfectants (PDPN-11, -14, and -17) was monitored using a whole-blood aggregometer following incubation with mouse whole blood as described in Materials and Methods. The aggregation reached a plateau within 20 minutes with the following aggregation amplitude: Mock: 2 ohms, PDPN-11, 6 ohms; PDPN-14, 8 ohms; PDPN-17, 40 ohms. Growth of the transfectants in monolayer culture using MTT assay (C) or in three-dimensional spheroid culture (D). Data are means \pm SEM of triplicate determinations. 
A

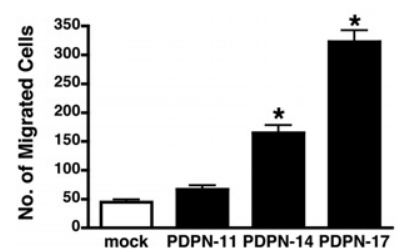

$\mathrm{B}$

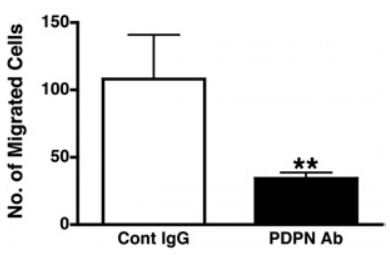

Figure 3. Podoplanin regulates cell migration in OS cell lines. A: Stable transfectants (PDPN-11, -14, and -17) or mock transfectants were seeded into transwell chambers and incubated for 18 hours in serum-free medium. The number of migrated cells was determined by counting stained cells in the lower well. ${ }^{*} P<0.001$ compared to mock transfectants by analysis of variance with the Tukey-Kramer test. B: U-2 OS cells were incubated with a control IgG (Co IgG) or an anti-podoplanin antibody (PDPN Ab) for 15 minutes before seeding into a transwell chamber as above. Data are means \pm SEM of triplicate determinations. ${ }^{* *} P=0.0022$ by the Mann-Whitney $U$-test.

tion-inducing activity, with the highest activity in PDPN-17 and the lowest in PDPN-11, corresponding to the exogenous podoplanin protein levels (Figure 2B), whereas the control mock-transfectant did not show platelet aggregation even after 1 hour (data not shown). Analysis of the growth rates of podoplanin-expressing clones demonstrated that they were not significantly different from that of the control cells in monolayer culture (Figure 2C). Likewise, three-dimensional spheroid growth did not show any differences in growth (Figure 2D). These findings further confirm that podoplanin functions as a platelet aggregation-inducing factor in OS cells but does not affect cell growth.

\section{Podoplanin Promotes OS Cell Migration}

To evaluate whether podoplanin overexpression causes altered migration behavior of OS cells, we used an in vitro migration assay. The transfectants showed a higher migration rate than mock cells; the migrated cell numbers of PDPN-17 and PDPN-14 were sixfold $(P<0.001)$ and threefold $(P<0.001)$ greater than that of mock cells, respectively, which was again proportional to the exogenous podoplanin levels (Figure 3A). PDPN-11, with the lowest expression level of exogenous podoplanin, showed an increase in migration, yet there was no statistical difference. To further confirm the increase in the motility of podoplanin-expressing cells, we tested whether or not blocking podoplanin function had any effect on motility. As shown in Figure 4B, U-2 OS cells neutralized with an anti-podoplanin antibody exhibited a significantly decreased migration, in comparison to the IgG-treated cells (108 \pm 33 versus $34 \pm 11, P=0.0022)$. These results indicate that podoplanin expression plays an important functional role in the migration of OS cells.

\section{c-Fos Up-Regulates Podoplanin Expression in Transformed Osteoblasts}

Since overexpression of c-Fos in transgenic mice results in the formation of OS, ${ }^{24}$ and since c-Fos was found to be up-regulated in a vast majority of human $\mathrm{OS}^{25}$ we next investigated its relevance to podoplanin expression in
OS. First, we investigated whether exogenous c-Fos affected the expression of podoplanin using a subclone of murine MC3T3-E1 osteoblastic cells, AT9.2, which expresses c-Fos in a tetracycline (Tet)-regulatable manner (Tet-off system). ${ }^{27}$ AT9.2 cells were cultured in the presence or the absence of Tet, and then semiquantitative RT-PCR analysis was performed to examine the expression of c-Fos, podoplanin, and an additional c-Fos target, TGF- $\beta 1$. Induction of exogenous c-Fos resulted in the stimulation of podoplanin expression as well as TGF- $\beta 1$ expression (Figure 4A). We then investigated podoplanin expression in the c-Fos transgenic OS-derived cell lines, $\mathrm{P} 1.7, \mathrm{P} 1.9$, and P1.15. ${ }^{24}$ Podoplanin was expressed in all
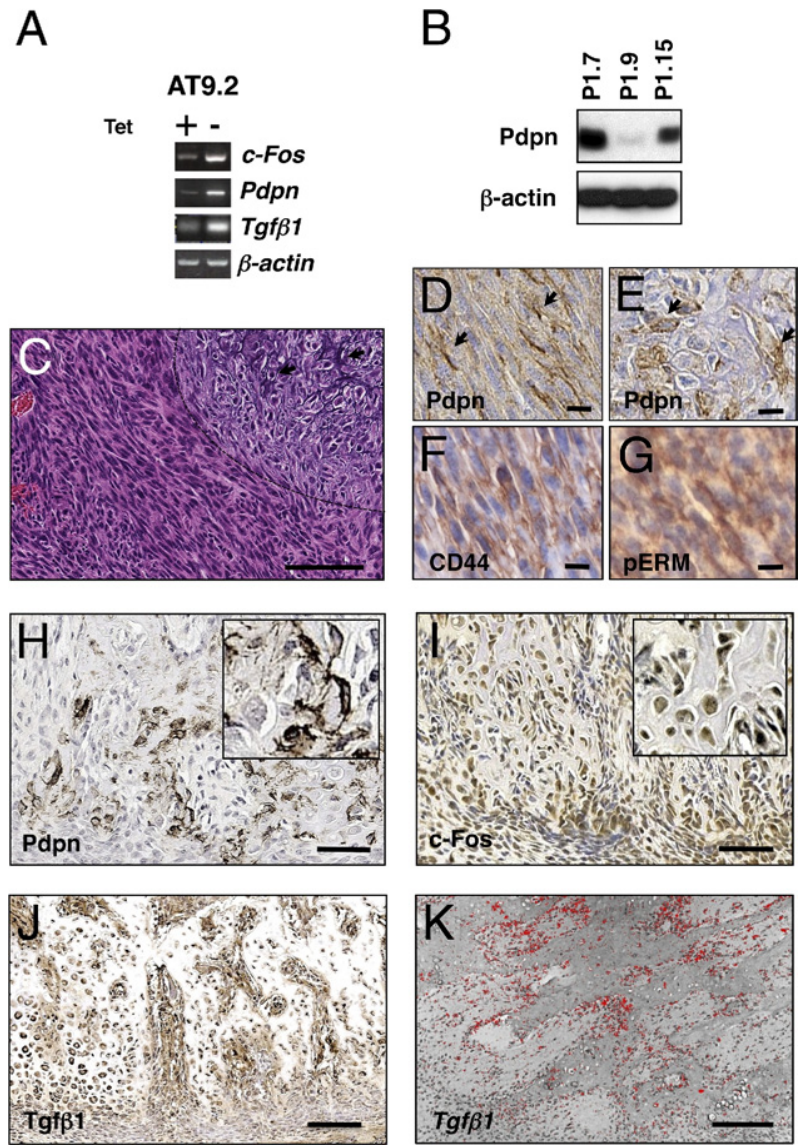

Figure 4. c-Fos-transformed osteoblasts induce podoplanin expression. A: c-Fos overexpression stimulates the expression of podoplanin (Pdpn) and Tgf $\beta 1$. Inducible c-Fos-overexpressing (Tet-off system) mouse osteoblasts (AT9. 2 cells) were cultured in the presence $(+)$ or absence $(-)$ of tetracycline (Tet), and then semiquantitative RT-PCR analysis was performed to examine c-Fos, podoplanin (Pdpn), and Tgf $\beta 1$ expression. $\beta$-actin was used as a loading control. B: Western blot analysis of podoplanin expression in c-Fos transgenic OS cells (P1.7, P1.9, P1.15) derived from c-Fos transgenic OS. C: Analysis of tumors induced in xenograft experiments using P1.15 cells. H\&E staining (top) showing the spindle tumor cells lie in a fascicle and an area of osteocartilagenous differentiation (demarcated by the dotted line; arrows indicate matrix with fine calcification). Immunohistochemistry using an antipodoplanin antibody shows specific cell surface staining in the spindle tumor cells (D, arrows) as well as in the cuboidal tumor cells (E, arrows); these cells were labeled for both CD 44 (F) and pERM (G). Immunohistochemistry analysis of c-Fos transgenic OS using antibodies against podoplanin (Pdpn, $\mathbf{H})$, c-Fos (I), and $\operatorname{Tgf} \beta 1$ (J). c-Fos transgenic OS showed a strong positivity for podoplanin in osteogenic tumor cells at the cell surface as well as at osteocyte-like dendritic processes within bone matrix. Radioactive in situ hybridization for Tgf $\beta 1$ mRNA showed strong signals within the tumor (K). Scale bars: $100 \mu \mathrm{m}(\mathbf{C}$ and $\mathbf{H}) ; 25 \mu \mathrm{m}(\mathbf{D}-\mathbf{G}) ; 200 \mu \mathrm{m}(\mathbf{I}-\mathbf{K})$ 
of the cell lines, albeit at different levels, as shown by Western blot analysis (Figure 4B). Moreover, subcutaneous tumor xenografts of these cells showed intense membranous podoplanin expression in elongated cells as well as in plump, cuboidal cells showing osteocartilagenous differentiation (Figure 4C). In addition, immunohistochemistry of tumor cells for CD44 and phosphorylated ERM (pERM) showed cell surface staining (Figure 4, $D-G)$.

To clarify in greater detail the cell types expressing podoplanin in vivo, immunohistochemistry was performed in normal mouse bone and c-Fos transgenic OS. In normal bone, podoplanin expression was observed in boneforming cells including osteocytes and osteoid osteocytes at their dendritic processes within canaliculi, as well as in very few mature bone-forming osteoblasts (only a few cells in an entire section) and periosteal cells (see Supplemental Figure S2 at http://ajp.amjpathol.org). In c-Fos transgenic mouse OS, osteoblastic tumor cells that were adjacent to bone matrix, as well as osteocyte-like tumor cells with irregular dendritic processes, showed high levels of podoplanin staining (Figure $4 \mathrm{H})$. All of the tumor cells were confirmed to express c-Fos protein (Figure $4 \mathrm{l}$ ). Moreover, high levels of TGF- $\beta 1$ protein and mRNA expression were observed in osteoblastic areas of c-Fos transgenic OS (Figure 4, J and K; see also Supplemental Figure S3 at http://ajp.amjpathol.org).

\section{Expression of Podoplanin in Human OS Tissues}

We carried out immunohistochemical analysis on normal bone and on OS TMA samples. In normal bone, osteoid osteocytes with abundant cytoplasm showed intense membrane immunoreactivity using a podoplanin antibody, notably at the spiky, urchin-like dendritic processes in the canaliculi, whereas mature osteocytes and surface-lining osteoblasts expressed little to no podoplanin (Figure 5A). We examined a total of 133 OS TMA samples, comprising 100 primary tumors and 33 metastatic lesions. Two distinct staining patterns were observed: First, in tumors that were undergoing so-called "normalization" or "maturation", ${ }^{34}$ tumor cells that were embedded in the excess of irregular neoplastic bone matrix showed an intense podoplanin staining pattern in the disorganized dendritic-like processes that was similar to osteoid osteocytes and C-Fos transgenic OS (Figure 5B). This was observed in $100 \%$ of cores from 51 primary and 15 metastatic tumors that were undergoing normalization (Table 1). Second, strong cell surface podoplanin staining was observed in pleomorphic and rounded tumor cells present in less differentiated tumors (Figure 5C). Interestingly, this was reminiscent of the expression pattern in c-Fos transgenic OS cell xenografts (Figure $4 C$ ). We next evaluated podoplanin expression across the different OS subtypes in both primary and metastatic tumors. From 80 osteoblastic OS (eg, Figure 5D), 65\% (52 of 80 ) showed membranous podoplanin staining, whereas 100\% (19 of 19) of chondroblastic OS (eg, Figure $5 \mathrm{E}$ ) and $79 \%$ (27 of 34) fibroblastic OS (eg, Figure 5F) expressed podoplanin at the cell membrane. Taken together, these results demonstrate high podoplanin ex-
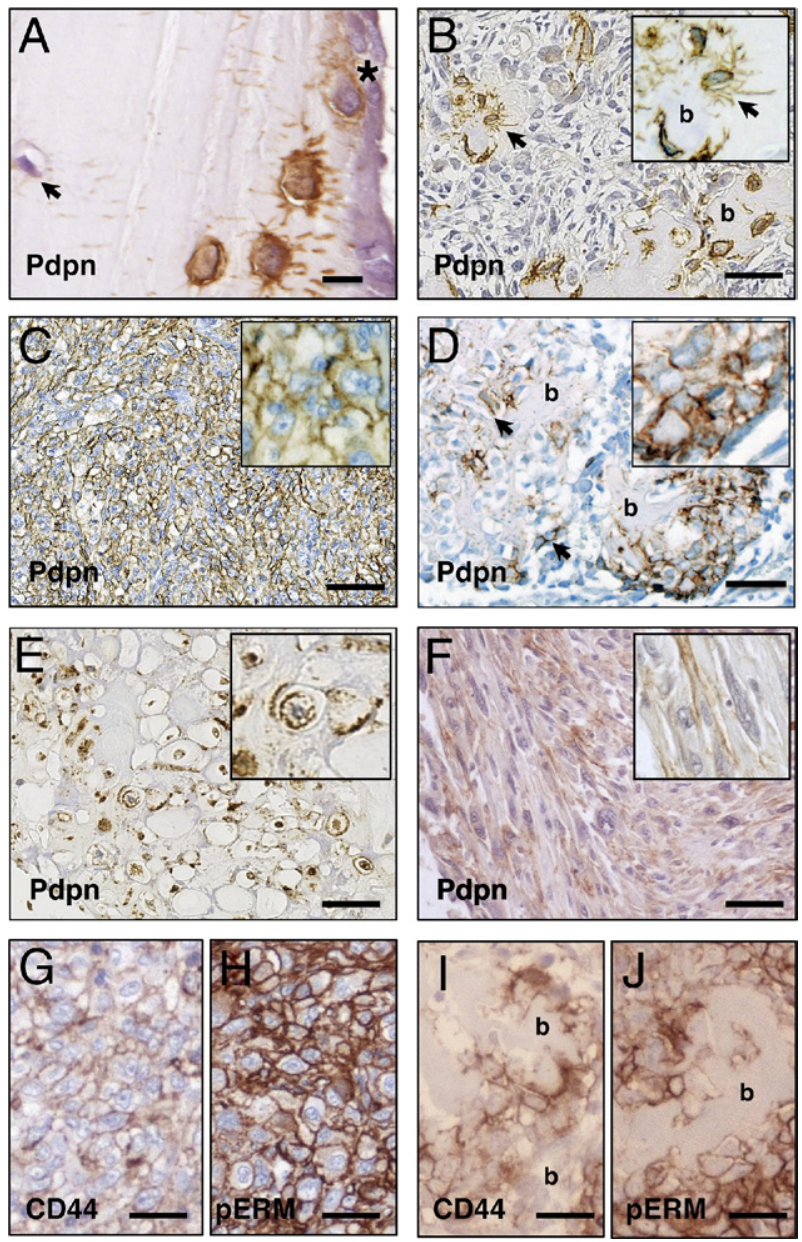

Figure 5. Podoplanin expression in human OS. Immunohistochemistry of podoplanin in normal human bone (A) and in human OS tissue (B-D). A Intense podoplanin labeling of osteoid osteocytes (immature osteocytes) and canaliculi in normal bone. Mature osteoblasts (asterisk) occasionally showed membrane staining, and mature osteocytes expressed podoplanin very weakly (arrow). B: Osteoblastic tumor cells in a more mature OS undergoing "normalization," also showing osteocytes embedded in irregularly shaped bone matrix. Osteocytes and osteocyte processes are clearly strongly positive for podoplanin (arrows). The insets show higher magnification of areas of interest. C: Undifferentiated OS with little extracellular matrix production. Pleomorphic and rounded tumor cells with rich cytoplasm showed intense membranous localization of podoplanin; D: Active osteoblastic component in an OS showing the typical malignant osteoid formation. Plump, cuboidal, osteoblastic tumor cells producing bone matrix (arrows) showed positive membrane staining for podoplanin, and occasionally showed antibody-labeled immature processes. E: Chondroblastic OS showing strong membranous staining for podoplanin. F: Fibroblastic OS with focal membranous immunoreactivity for podoplanin. $\mathbf{G}$ and $\mathbf{H}, \mathbf{I}$ and $\mathbf{J}$ : Immunostaining of the same tumors shown in $\mathbf{C}$ and $\mathbf{D}$, respectively, for CD44 (G and $\mathbf{I})$ and pERM (H and $\mathbf{J})$ showing membranous immunoreactivity of both CD44 and pERM. b, bone. Scale bars: $20 \mu \mathrm{m}(\mathbf{A}) ; 100 \mu \mathrm{m}(\mathbf{B}-\mathbf{F})$; $50 \mu \mathrm{m}(\mathbf{G}-\mathbf{J})$.

pression in the majority of human OS. In fact, there was an overall tendency toward metastatic OS samples expressing podoplanin at higher frequency than primary tumor samples, representing $87.9 \%$ (29 of 33 cores) versus $69 \%$ (69 of 100 cores), respectively. Finally, similarly to the mouse OS xenografts, immunolocalization studies showed cell surface expression of CD44 and pERM in human OS (Figure 5, G-J).

To further quantify and confirm podoplanin expression in human primary and metastatic OS, we performed real- 
A

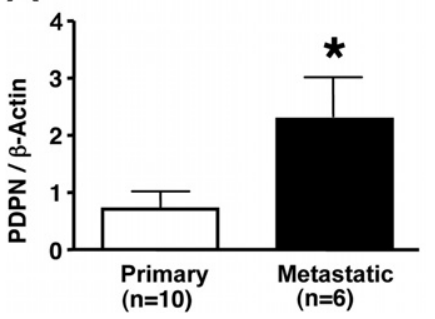

B

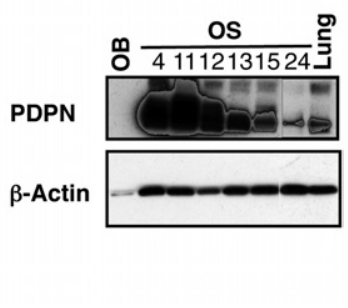

Figure 6. Podoplanin mRNA and protein expression in human OS. A: Comparison of the expression levels of podoplanin mRNA between 10 primary and six metastatic OS samples using real-time quantitative PCR analysis. Data represent the means \pm SEM of triplicate determinations. ${ }^{*} P=0.0312$. B: Western blot analysis of podoplanin protein expression from six OS samples. Normal lung (Lung) and normal human osteoblast (OB) lysates were used as controls.

time quantitative PCR studies and showed that the expression level of podoplanin mRNA in metastatic tumors was two- to threefold higher than in primary tumors $(P=$ 0.0312 , Figure 6A). Since the metastatic tumor samples also contained lung tissue, where type I alveolar cells are also known to express podoplanin, ${ }^{8}$ we examined podoplanin protein expression in normal lung tissue and normal primary osteoblasts for comparison. The data confirmed that the contribution of podoplanin expression from lung tissue was minimal (Figure 6B). Although we cannot rule out that lung tissue itself might induce podoplanin expression in the metastatic OS cells, our data suggest that the high expression levels of podoplanin protein in OS metastatic samples are largely due to OSderived podoplanin.

\section{Discussion}

In the current study, we have investigated the function and regulation of podoplanin in OS cells and have characterized its detailed expression pattern in both human OS as well as in c-Fos-induced OS in transgenic mice. We originally identified podoplanin as the platelet aggregation factor Aggrus, ${ }^{6}$ and demonstrated that podoplanin contributes to the establishment of tumor metastasis by promoting platelet aggregation. ${ }^{14}$ In the present study, we have extended these observations to OS, which show a high propensity to form lung metastases, and showed that podoplanin was expressed in three different human OS cell lines, which correlated with their ability to induce platelet aggregation. Indeed, functional studies showed that the platelet aggregation-inducing capacity was reduced by knockdown of endogenous podoplanin in human OS cells, and conversely, was promoted by overexpressing podoplanin in the nonmetastatic Dunn mouse OS cell line. Taken together, these results suggested that podoplanin plays an important functional role in a platelet aggregation during the process of OS metastasis.

Podoplanin was found previously to induce cell migration by modulating the actin cytoskeleton. ${ }^{13}$ In the present study, knockdown of endogenous podoplanin using siRNA resulted in reduced cell migration in OS cells. Moreover, a neutralizing anti-podoplanin antibody, which dramatically reduced tumor metastasis in our previous study, ${ }^{35}$ significantly reduced the motility of U-2 OS cells. One essential step in the sequences required for cell migration is filopodia formation mediated by Ezrin, a member of ERM (Ezrin-Radixin-Moesin) cytoskeleton-associated protein family, ${ }^{36}$ whose phosphorylation has been shown to be enhanced following podoplanin overexpression in MDCK and MCF7 cells. ${ }^{12,13}$ In addition, phosphorylated ERM proteins ( $\mathrm{EERM}$ ) directly link actin filaments and CD44, a glycoprotein receptor at the cell membrane..$^{37,38}$ In our study, we have shown in both mouse and human OS, that podoplanin is coexpressed with CD44 and pERM. This is supported by evidence confirming that podoplanin physically associates with CD44, ${ }^{39}$ and this interaction is crucial for podoplaninmediated cell migration. ${ }^{40}$ Furthermore, podoplanin is reported to interact directly with Ezrin, which triggers downstream activation of RhoA to regulate actin cytoskeletal dynamics. ${ }^{41}$ Ezrin is known to be a causative protein for OS metastasis, ${ }^{42}$ and our study showed that podoplanin expression levels in metastatic OS were higher than in primary OS. As we have shown coexpression of podoplanin/CD44/pERM, it is tempting to speculate that such a complex plays a role in the underlying mechanisms of cell migration and in further establishing metastasis in OS.

Podoplanin was up-regulated by the induction of the AP-1 oncoprotein, c-Fos, in mouse osteoblastic cells, which is consistent with the high expression of podoplanin in c-Fos-transformed OS and its derived OS cells. c-Fos is highly expressed in the vast majority of human $\mathrm{OS}^{25}$ as well as in most of OS cell lines and its viral counterpart, v-Fos, up-regulates several key molecules in tumorigenesis/metastasis, including Ezrin. ${ }^{42,43}$ Interestingly, Durchdewald et al recently reported that podoplanin is a novel c-Fos/AP-1 target gene. ${ }^{19} \mathrm{c}$-Fos physically binds to podoplanin promoter sequences, controls podoplanin transcription, and most importantly, plays a causative role in skin cancer development. ${ }^{19}$ We also revealed that TGF- $\beta 1$, which is a known activator of the AP- 1 transcription factor, ${ }^{44}$ is itself regulated by AP- $1,{ }^{45}$ and regulates podoplanin expression, ${ }^{12,46}$ was highly expressed in c-Fos transgenic mouse OS at both the mRNA and protein levels. Furthermore, nontransformed, c-Fosoverexpressing mouse osteoblasts showed high levels of podoplanin and TGF- $\beta 1$ expression in a C-Fos-dependent manner. Taken together, both $\mathrm{c}$-Fos and TGF- $\beta 1$ appear to act in concert and are very likely responsible for the high levels of podoplanin expression observed in c-Fos transgenic OS and human OS. These high podoplanin levels are an important observation because podoplanin expression is normally very tightly regulated and is expressed by only a few normal osteoblasts with no dendritic processes that are destined to become osteocytes. ${ }^{16}$ Thus, on the basis of the data presented here, we would suggest that the highly deregulated expression of podoplanin in OS contributes to OS tumor progression and metastasis.

In this study, we showed that podoplanin is expressed in more than two-thirds of conventional OS TMA samples. Consistent with our mRNA and immunoblot protein expression data, our immunohistochemical analysis of human OS revealed high expression of podoplanin in im- 
mature tumor cells with cell membrane localization, yet the number of positive lesional cells varied, presumably due to tumor heterogeneity and TMA core sampling variations. Meanwhile, virtually all of the tumor cells showing "normalization" or "maturation," which are characterized as a differentiation toward mature osteocyte-like cells in excessive bone matrix, ${ }^{34}$ expressed podoplanin at disorganized dendritic processes. These apparently paradoxical observations, that both immature and differentiating tumor cells express podoplanin, suggest that there is a podoplanin-positive immature subpopulation, which represents aggressive tumor cells, similar to the previously reported subpopulation of podoplanin-positive cells in the A431 human epidermoid carcinoma cell line. ${ }^{47}$ In addition, immature human OS cell lines such as MG-63, but not more mature human OS cells like Saos-2, and two cases of small cell osteosarcoma, which is a rare subtype of OS, ${ }^{34}$ are reported to show the same membranous immunohistochemical staining. ${ }^{7}$ Moreover, whereas c-Fos transgenic OS in vivo rarely showed strong specific membranous podoplanin antibody labeling, c-Fos transgenic OS-derived cell lines and their resulting tumor xenografts showed an intensive membranous podoplanin immunostaining. This further supports our notion that the normally tight control of podoplanin expression in normal bone appears to be lost in immature tumor cells.

Although podoplanin is a well-characterized osteocyte marker, its expression and function in bone neoplasms is not well understood. ${ }^{7}$ OS are known to express aberrantly a wide range of osteoblast differentiation markers, including transcription factors, serum enzymes such as alkaline phosphatase, noncollagenous bone proteins, and cell surface antigens. ${ }^{48}$ As tumor cells in OS are generally regarded as being immature, previous studies have focused typically on analyzing marker genes of early osteoblast differentiation, rather than late stage and fully differentiated osteoblast phenotypes. Thus, the elucidation of podoplanin expression across a wide range of different OS is important. We are currently continuing to investigate aberrant bone cell marker gene expression during the osteoblast differentiation program in different bone tumors including OS, which would be essential for understanding the pathobiology and for contributing further to an accurate histopathological diagnosis and a clinical management of bone tumors.

In conclusion, we have shown that podoplanin expression is under AP-1 control in osteoblasts and plays a crucial role in enhancing platelet aggregation and cell migration of OS. Thus, podoplanin and its regulator c-Fos could be a potential new therapeutic target in OS patients for preventing OS metastasis by attenuating cell migration as well as platelet aggregation.

\section{Acknowledgments}

We thank Dr. Takashi Nishiyama, Arisa Ogiwara, and Harumi Yamamura for their technical assistance; Prof. Gerhard Christofori (University of Basel, Switzerland) for critically reading the manuscript; and Prof. Nicolas A.
Athanasou (University of Oxford, UK) for helpful discussions.

\section{References}

1. Kempf-Bielack B, Bielack SS, Jurgens H, Branscheid D, Berdel WE Exner GU, Gobel U, Helmke K, Jundt G, Kabisch $H$, Kevric M, Klingebiel T, Kotz R, Maas R, Schwarz R, Semik M, Treuner J, Zoubek A, Winkler K: Osteosarcoma relapse after combined modality therapy: an analysis of unselected patients in the Cooperative Osteosarcoma Study Group (COSS). J Clin Oncol 2005, 23:559-568

2. Hayden JB, Hoang BH: Osteosarcoma: basic science and clinical implications. Orthop Clin North Am 2006, 37:1-7

3. Gasic GJ, Gasic TB, Galanti N, Johnson T, Murphy S: Platelet-tumorcell interactions in mice. The role of platelets in the spread of malignant disease, Int J Cancer 1973, 11:704-718

4. Mehta P: Potential role of platelets in the pathogenesis of tumor metastasis. Blood 1984, 63:55-63

5. Sugimoto $Y$, Watanabe $M$, Oh-hara T, Sato $S$, Isoe $T$, Tsuruo $T$ : Suppression of experimental lung colonization of a metastatic variant of murine colon adenocarcinoma 26 by a monoclonal antibody $8 \mathrm{~F} 11$ inhibiting tumor cell-induced platelet aggregation. Cancer Res 1991, 51:921-925

6. Kato Y, Fujita N, Kunita A, Sato S, Kaneko M, Osawa M, Tsuruo T: Molecular identification of Aggrus/T1alpha as a platelet aggregationinducing factor expressed in colorectal tumors. J Biol Chem 2003, 278:51599-51605

7. Ariizumi T, Ogose A, Kawashima H, Hotta T, Li G, Xu Y, Umezu H, Sugai $\mathrm{M}$, Endo $\mathrm{N}$ : Expression of podoplanin in human bone and bone tumors: new marker of osteogenic and chondrogenic bone tumors. Pathol Int 2010, 60:193-202

8. Schacht V, Dadras SS, Johnson LA, Jackson DG, Hong YK, Detmar M: Up-regulation of the lymphatic marker podoplanin, a mucin-type transmembrane glycoprotein, in human squamous cell carcinomas and germ cell tumors. Am J Pathol 2005, 166:913-921

9. Wicki A, Christofori G: The potential role of podoplanin in tumour invasion. Br J Cancer 2007, 96:1-5

10. Chuang WY, Yeh CJ, Wu YC, Chao YK, Liu YH, Tseng CK, Chang HK, Liu HP, Hsueh C: Tumor cell expression of podoplanin correlates with nodal metastasis in esophageal squamous cell carcinoma. Histol Histopathol 2009, 24:1021-1027

11. Kreppel M, Scheer M, Drebber U, Ritter L, Zoller JE: Impact of podoplanin expression in oral squamous cell carcinoma: clinical and histopathologic correlations. Virchows Arch 2010, 456:473-482

12. Wicki A, Lehembre F, Wick N, Hantusch B, Kerjaschki D, Christofori G: Tumor invasion in the absence of epithelial-mesenchymal transition: podoplanin-mediated remodeling of the actin cytoskeleton. Cancer Cell 2006, 9:261-272

13. Martin-Villar E, Megias D, Castel S, Yurrita MM, Vilaro S, Quintanilla M: Podoplanin binds ERM proteins to activate RhoA and promote epithelial-mesenchymal transition. J Cell Sci 2006, 119:4541-4553

14. Kunita A, Kashima TG, Morishita Y, Fukayama M, Kato Y, Tsuruo T, Fujita N: The platelet aggregation-inducing factor aggrus/podoplanin promotes pulmonary metastasis. Am J Pathol 2007, 170:1337-1347

15. Edwards JR, Williams K, Kindblom LG, Meis-Kindblom JM, Hogendoorn PC, Hughes D, Forsyth RG, Jackson D, Athanasou NA: Lymphatics and bone. Hum Pathol 2008, 39:49-55

16. Guo D, Bonewald LF: Advancing our understanding of osteocyte cell biology. Ther Adv Musculoskel Dis 2009, 1:87-96

17. Hantusch B, Kalt R, Krieger S, Puri C, Kerjaschki D: Sp1/Sp3 and DNA-methylation contribute to basal transcriptional activation of human podoplanin in MG63 versus Saos-2 osteoblastic cells. BMC Mol Biol 2007, 8:20

18. Shen Y, Chen CS, Ichikawa H, Goldberg GS: SRC induces podoplanin expression to promote cell migration. J Biol Chem 2010, 285 : 9649-9656

19. Durchdewald M, Guinea-Viniegra J, Haag D, Riehl A, Lichter P, Hahn M, Wagner EF, Angel P, Hess J: Podoplanin is a novel fos target gene in skin carcinogenesis. Cancer Res 2008, 68:6877-6883

20. Angel P, Karin M: The role of Jun. Fos and the AP-1 complex in cell-proliferation and transformation, Biochim Biophys Acta 1991, 1072:129-157 
21. Wagner EF: AP-1-Introductory remarks. Oncogene 2001, 20:23342335

22. Eferl R, Wagner EF: AP-1: a double-edged sword in tumorigenesis Nat Rev Cancer 2003, 3:859-868

23. Zenz R, Eferl R, Scheinecker C, Redlich K, Smolen J, Schonthaler HB, Kenner L, Tschachler E, Wagner EF: Activator protein 1 (Fos/Jun) functions in inflammatory bone and skin disease. Arthritis Res Ther 2008, 10:201

24. Grigoriadis AE, Schellander K, Wang ZQ, Wagner EF: Osteoblasts are target cells for transformation in c-fos transgenic mice. J Cell Biol 1993, 122:685-701

25. Papachristou DJ, Batistatou A, Sykiotis GP, Varakis I, Papavassiliou AG: Activation of the JNK-AP-1 signal transduction pathway is associated with pathogenesis and progression of human osteosarcomas. Bone 2003, 32:364-371

26. Mehta P, Lawson D, Ward MB, Kimura A, Gee A: Effect of human tumor cells on platelet aggregation: potential relevance to pattern of metastasis. Cancer Res 1987, 47:3115-3117

27. Sunters A, Thomas DP, Yeudall WA, Grigoriadis AE: Accelerated cel cycle progression in osteoblasts overexpressing the c-fos protooncogene: induction of cyclin A and enhanced CDK2 activity. J Biol Chem 2004, 279:9882-9891

28. Asai T, Ueda T, Itoh K, Yoshioka K, Aoki Y, Mori S, Yoshikawa H: Establishment and characterization of a murine osteosarcoma cell line (LM8) with high metastatic potential to the lung. Int J Cancer 1998, 76:418-22

29. Kashima T, Kawaguchi J, Takeshita S, Kuroda M, Takanashi M, Horiuchi H, Imamura T, Ishikawa Y, Ishida T, Mori S, Machinami R, Kudo A: Anomalous cadherin expression in osteosarcoma. Possible relationships to metastasis and morphogenesis, Am J Pathol 1999, 155:1549-1555

30. Ogasawara S, Kaneko MK, Price JE, Kato Y: Characterization of anti-podoplanin monoclonal antibodies: critical epitopes for neutralizing the interaction between podoplanin and CLEC-2. Hybridoma (Larchmt) 2008, 27:259-267

31. Kashima T, Nakamura K, Kawaguchi J, Takanashi M, Ishida T, Aburatani $H$, Kudo A, Fukayama M, Grigoriadis AE: Overexpression of cadherins suppresses pulmonary metastasis of osteosarcoma in vivo. Int J Cancer 2003, 104:147-154

32. Cardinal DC, Flower RJ: The electronic aggregometer: a novel device for assessing platelet behavior in blood. J Pharmacol 1980, 3:135-158

33. Ohazama A, Johnson EB, Ota MS, Choi HY, Porntaveetus T, Oommen S, Itoh N, Eto K, Gritli-Linde A, Herz J, Sharpe PT: Lrp4 modulates extracellular integration of cell signaling pathways in development. PLoS One 2008, 3:e4092

34. Klein MJ, Siegal GP: Osteosarcoma: anatomic and histologic variants. Am J Clin Pathol 2006, 125:555-581
35. Kato Y, Kaneko MK, Kunita A, Ito H, Kameyama A, Ogasawara S, Matsuura N, Hasegawa Y, Suzuki-Inoue K, Inoue O, Ozaki Y, Narimatsu $\mathrm{H}$ : Molecular analysis of the pathophysiological binding of the platelet aggregation-inducing factor podoplanin to the C-type lectinlike receptor CLEC-2. Cancer Sci 2008, 99:54-61

36. Hunter KW: Ezrin, a key component in tumor metastasis. Trends Mol Med 2004, 10:201-204

37. Tsukita S, Yonemura S: ERM (ezrin/radixin/moesin) family: from cytoskeleton to signal transduction. Curr Opin Cell Biol 1997, 9:70-75

38. Yonemura S, Hirao M, Doi Y, Takahashi N, Kondo T, Tsukita S, Tsukita S: Ezrin/radixin/moesin (ERM) proteins bind to a positively charged amino acid cluster in the juxta-membrane cytoplasmic domain of CD44. CD43, and ICAM-2, J Cell Biol 1998, 140:885-895

39. Ohizumi I, Harada N, Taniguchi K, Tsutsumi Y, Nakagawa S, Kaiho S, Mayumi T: Association of CD44 with OTS-8 in tumor vascular endothelial cells. Biochim Biophys Acta 2000, 1497:197-203

40. Martin-Villar E, Fernandez-Munoz B, Parsons M, Yurrita MM, Megias D, Perez-Gomez E, Jones GE, Quintanilla M: Podoplanin associates with CD44 to promote directional cell migration. Mol Biol Cell 2010, 21:4387-4399

41. Scholl FG, Gamallo C, Vilaro S, Quintanilla M: Identification of PA2.26 antigen as a novel cell-surface mucin-type glycoprotein that induces plasma membrane extensions and increased motility in keratinocytes. J Cell Sci 1999, 112:4601-4613

42. Khanna C, Wan X, Bose S, Cassaday R, Olomu O, Mendoza A, Yeung C, Gorlick R, Hewitt SM, Helman LJ: The membrane-cytoskeleton linker ezrin is necessary for osteosarcoma metastasis. Nat Med 2004 10:182-186

43. Ozanne BW, Spence HJ, McGarry LC, Hennigan RF: Transcription factors control invasion: aP-1 the first among equals. Oncogene 2007, 26:1-10

44. Uria JA, Jimenez MG, Balbin M, Freije JM, Lopez-Otin C: Differential effects of transforming growth factor-beta on the expression of collagenase-1 and collagenase-3 in human fibroblasts. J Biol Chem 1998, 273:9769-9777

45. Kim SJ, Angel P, Lafyatis R, Hattori K, Kim KY, Sporn MB, Karin M, Roberts $A B$ : Autoinduction of transforming growth factor beta 1 is mediated by the AP-1 complex. Mol Cell Biol 1990, 10:1492-1497

46. Suzuki H, Kato Y, Kaneko MK, Okita Y, Narimatsu H, Kato M: Induction of podoplanin by transforming growth factor-beta in human fibrosarcoma. FEBS Lett 2008, 582:341-345

47. Atsumi N, Ishii G, Kojima M, Sanada M, Fujii S, Ochiai A: Podoplanin, a novel marker of tumor-initiating cells in human squamous cell carcinoma A431. Biochem Biophys Res Commun 2008, 373:36-41

48. Tang N, Song WX, Luo J, Haydon RC, He TC: Osteosarcoma development and stem cell differentiation. Clin Orthop Relat Res 2008 , 466:2114-2130 